\title{
The Novel Rational Spectral Collocation Method for a Singularly Perturbed Reaction-Diffusion Problem with Nonsmooth Data
}

\author{
Lufeng Yang $\mathbb{D}^{1,2}$ \\ ${ }^{1}$ School of Mathematics and Information Science, North Minzu University, Yinchuan 750021, China \\ ${ }^{2}$ School of Mathematics and Statistics, Lanzhou University, Lanzhou 730000, China \\ Correspondence should be addressed to Lufeng Yang; ylf-sd@163.com
}

Received 27 August 2020; Revised 30 January 2021; Accepted 15 February 2021; Published 26 February 2021

Academic Editor: Fotios Georgiades

Copyright $(02021$ Lufeng Yang. This is an open access article distributed under the Creative Commons Attribution License, which permits unrestricted use, distribution, and reproduction in any medium, provided the original work is properly cited.

A singularly perturbed reaction-diffusion problem with a discontinuous source term is considered. A novel rational spectral collocation combined with a singularity-separated method for this problem is presented. The solution is expressed as $u=w+v$, where $w$ is the solution of corresponding auxiliary boundary value problem and $v$ is a singular correction with direct expressions. The rational spectral collocation method combined with a sinh transformation is applied to solve the weakened singularly boundary value problem. According to the asymptotic analysis, the sinh transformation parameters can be determined by the width and position of the boundary layers. The parameters in the singular correction can be determined by the boundary conditions of the original problem. Numerical experiment supports theoretical results and shows that compared with previous research results, the novel method has the advantages of a high computational accuracy in singularly perturbed reaction-diffusion problems with nonsmooth data.

\section{Introduction}

Singular perturbation problems arise in mathematical modeling of physical and engineering problems, such as the boundary layer of fluid mechanics, the turning point of quantum mechanics, and the flow of large Reynolds numbers. In recent decades, the singular perturbation problem has received extensive attention. Detailed theories and analysis of the singular perturbation problem can be found in the literature $[1,2]$. These problems have steep gradients in narrow layers, which is a serious obstacle in calculating classical numerical methods.

Many studies of singularly perturbed problems with nonsmooth data have been researched. The first-order Schwarz method with uniform parameters on Shishkin's mesh was proposed for a singularly perturbed reaction-diffusion problem with a nonsmooth source term [3].Chandru and Shanthi applied a boundary value technique and hybrid difference scheme for singularly perturbed boundary value problem of reactiondiffusion type with discontinuous source term in $[4,5]$, respectively. The second-order finite element method was presented on a Bakhvalov-Shishkin's mesh for singularly perturbed problems with the interior layer [6]. A uniformly convergent difference scheme was employed for singularly perturbed semilinear problems with a discontinuous source term and proved that this scheme was the first-order convergence [7]. A uniformly numerical convergence scheme based on piecewiseuniform Shishkin's meshes was developed for a reaction-diffusion equation with a discontinuous diffusion coefficient and proved to have second-order convergence [8]. A hybrid difference scheme on a Shishkin's mesh was introduced for a singularly perturbed reaction-diffusion problem with one and two parameters [9] with interior layers.

The second-order singularly perturbed reaction-diffusion boundary value problem with discontinuous source term is considered: 


$$
\left\{\begin{array}{l}
L u(x):=-\varepsilon u^{\prime \prime}+c u=f(x), \quad x \in \Omega^{-} \cup \Omega^{+}, \\
u(0)=\alpha, u(1)=\beta
\end{array}\right.
$$

where $0<\varepsilon \ll 1$ is a small positive parameter, $c>0$. Assuming the function $f(x)$ is sufficiently smooth in $\Omega$, a jump discontinuity is expected at $d \in \Omega$ denoted by $[w](d)=w\left(d^{+}\right)-w\left(d^{-}\right)$. In general, this discontinuity of $f$ gives rise to interior layers in the first derivative of the exact solution. Here, $\Omega^{-}=(0, d), \Omega^{+}=(d, 1)$.

The rational spectral collocation method was proposed in the literature [10]. A conformal map maps the collocation points clustered near the poles of $[-1,1]$, which means more points are near the poles. The parameters of the mapping depend on the position and width of the boundary layer. A rational spectral collocation in a barycentric form with sinh transform (RSC-sinh method) is applied to solve a coupled system of singularly perturbed problems and third-order singularly perturbed problems $[11,12]$.

To weaken the singularity and improve the accuracy of numerical simulation, the singularity-separated method (SSM) for the singular perturbation problem with constant coefficients was proposed by Chen and Yang [13]. Finite element methods with SSM were used to solve a singular perturbation problem with a single boundary layer. A novel rational spectral collocation method is presented combined with the singularity-separated technique for a system of singularly perturbed boundary value problems [14].

A novel numerical method based on the rational spectral collocation in a barycentric form with a singularly-separated method (RSC-SSM) is proposed for solving the secondorder singularly perturbed reaction-diffusion problem with nonsmooth source term.

This paper is organized as follows. The asymptotic analysis and sinh transform is outlined in Section 2. The algorithmic details of RSC-SSM for second-order singularly perturbed boundary value problem are considered, and the error estimates for the method are discussed in Section 3. The singularly perturbed reaction-diffusion problem with discontinuous source term is solved in Section 4, which supports theoretical results and provides a favorable comparison with existing methods. Finally, the conclusions are drawn in Section 5.

\section{Preliminaries}

In this section, some useful lemmas are given, which provide information about the boundary and interior layers occurring in the solution of singularly perturbed problem (1).
Theorem 1. The problem (1) has a solution $u \in C^{1}(\Omega) \cap C^{2}\left(\Omega^{-} \cup \Omega^{+}\right)$.

Proof. This theorem is proved constructively. Let $u_{1}$ and $u_{2}$ be particular solutions of the following differential equations, respectively:

$$
\begin{array}{ll}
-\varepsilon u_{1}^{\prime \prime}+b(x) u_{1}=f^{-}(x), & x \in \Omega^{-}, \\
-\varepsilon u_{2}^{\prime \prime}+b(x) u_{2}=f^{+}(x), & x \in \Omega^{+} .
\end{array}
$$

Consider the function

$$
u(x)= \begin{cases}u_{1}(x)+\left(u(0)-u_{1}(0)\right) \phi_{1}(x)+A \phi_{2}(x), & x \in \Omega^{-}, \\ u_{2}(x)+B \phi_{1}(x)+\left(u(1)-u_{2}(1)\right) \phi_{2}(x), & x \in \Omega^{+},\end{cases}
$$

where $\phi_{1}(x)$ and $\phi_{2}(x)$ are the solutions of the boundary value problems:

$$
\begin{aligned}
& -\varepsilon \phi_{1}^{\prime \prime}+b(x) \phi_{1}=0, \quad x \in \Omega, \phi_{1}(0)=1, \phi_{1}(1)=0, \\
& -\varepsilon \phi_{2}^{\prime \prime}+b(x) \phi_{2}=0, \quad x \in \Omega, \phi_{2}(0)=0, \phi_{2}(1)=1,
\end{aligned}
$$

and $A$ and $B$ are constants to be chosen so that $u \in C^{1}(\Omega)$. Using the fact that the maximum principle states that the maximum and minimum values taken on by $\phi_{i} \in \bar{\Omega}, \quad i=1,2$ must occur on $\partial \Omega$, then we have $0<\phi_{i}<1, i=1,2$, and

$$
\begin{aligned}
& \phi_{1}^{\prime}<0, \\
& \phi_{2}^{\prime}>0, \\
& x \in \Omega .
\end{aligned}
$$

We need to choose the constants $A$ and $B$ so that $u \in C^{1}(\Omega)$; then, we impose

$$
u\left(d^{-}\right)=u\left(d^{+}\right), u^{\prime}\left(d^{-}\right)=u^{\prime}\left(d^{+}\right) .
$$

Additionally, the constants A and B satisfy

$$
\left\{\begin{array}{l}
A \phi_{2}\left(d^{-}\right)-B \phi_{1}\left(d^{+}\right)=g^{(0)} \\
A \phi_{2}^{\prime}\left(d^{-}\right)-B \phi_{1}^{\prime}\left(d^{+}\right)=g^{(1)}
\end{array}\right.
$$

where

$$
\begin{aligned}
g^{(i)}= & u_{2}^{(i)}\left(d^{+}\right)-u_{1}^{(i)}\left(d^{-}\right)+\left(u(1)-u_{2}(1)\right) \phi_{2}^{(i)}\left(d^{+}\right) \\
& -\left(u(0)-u_{1}(0)\right) \phi_{1}^{(i)}\left(d^{-}\right), \quad i=0,1 .
\end{aligned}
$$

Since

$\left|\begin{array}{lr}\phi_{2}\left(d^{-}\right) & -\phi_{1}\left(d^{+}\right) \\ \phi_{2}^{\prime}\left(d^{-}\right) & -\phi_{1}^{\prime}\left(d^{+}\right)\end{array}\right|=-\phi_{1}^{\prime}\left(d^{+}\right) \phi_{2}\left(d^{-}\right)+\phi_{1}\left(d^{+}\right) \phi_{2}^{\prime}\left(d^{-}\right)>0$,

the system of linear equation (7) has a solution.

Lemma 1. Let us suppose that a function $u(x)$ satisfies $u(0) \geq 0, u(1) \geq 0, L u(x) \geq 0, \forall x \in \Omega^{-} \cup \Omega^{+}$and $u[d]=0$, $\left[u^{\prime}\right](d) \leq 0$, then $u(x) \geq 0, \forall x \in \bar{\Omega}$.

Proof. Let $x_{0}$ be such that $u\left(x_{0}\right)=\min _{\Omega} u(x)$. If $u\left(x_{0}\right)>0$, the conclusion is clearly correct. Suppose that $u\left(x_{0}\right)<0$; it is clear that either $x_{0} \in \Omega^{-} \cup \Omega^{+}$or $x_{0}=d$. In the first case, $u^{\prime}\left(x_{0}\right)=0, u^{\prime \prime}\left(x_{0}\right) \geq 0$ and

$$
L u\left(x_{0}\right)=-\varepsilon u^{\prime \prime}\left(x_{0}\right)+c u\left(x_{0}\right)<0,
$$


which is a contradiction. In the second case, the proof depends on whether or not $u$ is differentiable at $d$. If $u$ is not differentiable at $d$, then $\left[u^{\prime}\right](d) \neq 0$. Since $u^{\prime}\left(d^{-}\right) \geq 0, u^{\prime}\left(d^{+}\right) \leq 0$, it is clear that $\left[u^{\prime}\right](d)>0$, which is a contradiction. If $u$ is differentiable at $d$, according to the mean value theorem, $u \in C^{1}(\bar{\Omega})$. Since $u(d)<0$, it follows that there exists a neighbourhood $\Omega_{h}=(d-h, d)$ such that $u(x)<0, \forall x \in \Omega_{h}$. Choose a point $x_{1} \neq d, x_{1} \in \Omega_{h}$ such that $u\left(x_{1}\right)>u(d)$. Then, the mean value theorem indicates that $\exists x_{2} \in\left(x_{1}, d\right):$

$$
u^{\prime}\left(x_{2}\right)=\frac{u(d)-u\left(x_{1}\right)}{d-x_{1}}<0,
$$

and similarly, there also exists $x_{3} \in \Omega_{h}$ :

$$
u^{\prime \prime}\left(x_{3}\right)=\frac{u^{\prime}(d)-u^{\prime}\left(x_{2}\right)}{d-x_{2}}=\frac{-u^{\prime}\left(x_{2}\right)}{d-x_{2}}>0 .
$$

Thus, we have

$$
L u\left(x_{3}\right)=-\varepsilon u^{\prime \prime}\left(x_{3}\right)+c u\left(x_{3}\right)<0,
$$

which is a contradiction.

The direct application of the maximum principle is the following stability result.

Theorem 2. Let $u(x)$ be the solution of (1), which can be estimated as

$$
\|u\|_{\bar{\Omega}} \leq \max \left(|\alpha|,|\beta|, \frac{|f(x)|}{b}\right) .
$$

Proof. Setting $M=\max (|\alpha|,|\beta|,(|f(x)| / b))$, we can construct $v=M \pm u$ satisfying

$$
\left\{\begin{array}{l}
L v=-\varepsilon v^{\prime \prime} \pm b v=b M \pm L u=b M \pm f \geq 0, \\
v(0)=M \pm \alpha \geq 0, v(1)=M \pm \beta \geq 0 .
\end{array}\right.
$$

Furthermore, the solution $u$ of (1) has first-order continuous differentiability, that is,

$$
\begin{aligned}
{[v](d) } & =[u](d)=0, \\
{\left[v^{\prime}\right](d) } & =[u]^{\prime}(d)=0 .
\end{aligned}
$$

It follows from the maximum principle that $v \geq 0$ for all $x \in \bar{\Omega}$; thus, we obtain

$$
\|u\|_{\Omega} \leq \max \left(|\alpha|,|\beta|, \frac{|f(x)|}{b}\right) .
$$

Theorem 3. The problem (1) has a unique solution $u \in C^{1}(\Omega) \cap C^{2}\left(\Omega^{-} \cup \Omega^{+}\right)$.
Proof. The existence of problem (1) has been proved in Theorem 1. Let $u_{1}(x), u_{2}(x)$ be the solution of problem (1), and $u_{1}(x) \neq u_{2}(x), \forall x \in[0,1]$; set $h(x)=u_{1}(x)-u_{2}(x)$. Then, we have

$$
\begin{aligned}
L h(x) & =L u_{1}(x)-L u_{2}(x)=f(x)-f(x)=0, \\
h(0) & =h(1)=0, \\
{[h](d) } & =\left[h^{\prime}\right](d)=0 .
\end{aligned}
$$

It follows at once from Theorem 2, and we can obtain $h(x)=0$.

Thus, problem (1) has a unique solution.

2.1. Asymptotic Expansion Approximation. For the construction of the RSC-SSM method, it is necessary to use the exact solution of the problem to analyze the position and width of the boundary layer. The problem (1) is considered as the coupling of the following two subproblems:

$$
\begin{aligned}
& P_{L}:\left\{\begin{array}{l}
L^{-} u(x)=-\varepsilon u^{\prime \prime}+c u=f^{-}(x), \quad x \in \Omega^{-}, \\
u(0)=\alpha, u\left(d^{-}\right)=\gamma,
\end{array}\right. \\
& P_{R}:\left\{\begin{array}{l}
L^{+} u(x)=-\varepsilon u^{\prime \prime}+c u=f^{+}(x), \quad x \in \Omega^{+}, \\
u\left(d^{+}\right)=\gamma, u(1)=\beta,
\end{array}\right.
\end{aligned}
$$

where $\gamma$ is the value of $u$ at $d$, which is determined by $u^{\prime}\left(d^{-}\right)=u^{\prime}\left(d^{+}\right)$.

The Shishkin decomposition method splits the solution of the singular perturbation problem (1) into regular $\omega$ and layer $v$ components. The regular component $\omega$ is defined as the solution of

$$
\left\{\begin{array}{l}
L \omega(x)=f(x), \quad x \in \Omega^{-} \cup \Omega^{+}, \\
\omega(0)=\frac{f^{-}(0)}{c}, \\
\omega\left(d^{-}\right)=\frac{f^{-}\left(d^{-}\right)}{c}, \\
\omega\left(d^{+}\right)=\frac{f^{+}\left(d^{+}\right)}{c}, \\
\omega(1)=\frac{f^{+}(1)}{c} .
\end{array}\right.
$$

The singular component $v$ is given by 


$$
\left\{\begin{array}{l}
L v(x)=f(x), \quad x \in \Omega^{-} \cup \Omega^{+} \\
{[v](d)=-[\omega](d)} \\
{\left[v^{\prime}\right](d)=-\left[\omega^{\prime}\right](d),} \\
v(0)=u(0)-\omega(0) \\
v(1)=u(1)-\omega(1)
\end{array}\right.
$$

Lemma 2. For each integer $k$ satisfying $0 \leq k \leq 4$, the regular term $\omega$ and the singular term $v$ satisfy the bounds

$$
\begin{aligned}
& |\omega(x)| \leq \begin{cases}C\left(1+\varepsilon^{1-(k / 2)} E_{1}(x)\right), & x \in \Omega^{-}, \\
C\left(1+\varepsilon^{1-(k / 2)} E_{2}(x)\right), & x \in \Omega^{+},\end{cases} \\
& |v(x)| \leq \begin{cases}C\left(\varepsilon^{-(k / 2)} E_{1}(x)\right), & x \in \Omega^{-}, \\
C\left(\varepsilon^{-(k / 2)} E_{2}(x)\right), & x \in \Omega^{+},\end{cases}
\end{aligned}
$$

where $C$ is a constant independent of $\varepsilon$ and

$$
\begin{aligned}
& E_{1}(x)=e^{-x \sqrt{(c / \varepsilon)}}+e^{-(d-x) \sqrt{(c / \varepsilon)}}, \\
& E_{2}(x)=e^{-(x-d) \sqrt{(c / \varepsilon)}}+e^{-(1-x) \sqrt{(c / \varepsilon)}} .
\end{aligned}
$$

Proof. This is found using the stability result in Theorem 2 and the techniques in [3].

Lemma 3 (see [12]). Considering the singularly perturbed reaction-diffusion

$$
\left\{\begin{array}{l}
-\varepsilon u^{\prime \prime}+c u=f(x), \quad x \in(0,1) \\
u(0)=\alpha \\
u(1)=\beta
\end{array}\right.
$$

where $f(x)$ is sufficiently smooth in $[0,1]$, the solution $u$ of this problem has the following asymptotic expansion:

$$
u(x)=w(x)+\bar{v}(x)+O(\sqrt{\varepsilon}),
$$

where

$$
\bar{v}(x)=\left\{\begin{array}{ll}
v(x), & x \in\left[0, \tau_{0}\right] \cup\left[1-\tau_{0}, 1\right], \\
0, & x \in\left[\tau_{0}, 1-\tau_{0}\right],
\end{array} \quad 0<\tau_{0}=-\frac{\sqrt{\varepsilon}}{c} \ln \sqrt{\varepsilon} \ll 1,\right.
$$

and the following inequality is satisfied:

$$
\|u-(w+\bar{v})\| \leq C \sqrt{\varepsilon},
$$

where $C$ is a generic constant.

Lemma 3 indicates that the boundary layer region of the singularly perturbed reaction-diffusion problem with smooth source term is $\left[0, \tau_{0}\right]$ and $\left[1-\tau_{0}, 1\right]$. In other words, the position of the boundary layer is at the two endpoints in the interval $[0,1]$, and its width is $\delta=\tau_{0}$.
2.2. Rational Spectral Collocation Method in the Barycentric Form. A rational interpolation function $p_{N}(x)$ in the barycentric form of a function $u(x)$ can be expressed as follows [15]:

$$
u(x) \approx p_{N}(x)=\frac{\sum_{k=0}^{N}\left(\omega_{k} /\left(x-x_{k}\right)\right) u\left(x_{k}\right)}{\sum_{k=0}^{N}\left(\omega_{k} / x-x_{k}\right)},
$$

where $\left\{\omega_{k}\right\}_{k=0}^{N}$ are barycentric weights and $\left\{x_{k}\right\}_{k=0}^{N}$ are distinct interpolation points. Most important in practice are the so-called Chebyshev-Gauss-Lobatto points $x_{k}=-\cos (k \pi / N), k=0,1, \ldots, N$, and in this case, the barycentric weights are as follows [15]:

$$
\begin{aligned}
& \omega_{0}=\frac{1}{2}, \\
& \omega_{k}=(-1)^{k}, \quad k=1,2, \ldots, N-1, \\
& \omega_{N}=\frac{(-1)^{N}}{2} .
\end{aligned}
$$

Theorem 4 (see [16]). Let $D_{1}$ and $D_{2}$ be domains in $C$ containing $J=[-1,1]$ and a real interval $I$, respectively. Let $g: D_{1} \longrightarrow D_{2}$ be a conformal map such that $g(J)=I$. If $f: D_{2} \longrightarrow C$ is such that $f \circ g: D_{1} \longrightarrow C$ is analytic inside and on an ellipse with foci \pm 1 , semimajor axis length $L$, and semiminor axis length $l$, then the rational function $p_{N}(x)$, which interpolates $f$ at the transformed Chebyshev points $\widehat{x}_{k}=g\left(x_{k}\right)$ satisfies

$$
\left|p_{N}(x)-f(x)\right|=O\left((L+l)^{-N}\right),
$$

uniformly for all $x \in[-1,1]$.

As suggested in (31), the convergence rate of the rational spectral collocation method mainly depends on the analytic region of $u$ in the complex plane. Thus, the conformal map $g$ could be chosen to enlarge the ellipse of analyticity of $u \circ g$. Then, compared with the Chebyshev spectral method, a better approximation of $u$ could be obtained.

An advantage of the rational function in barycentric form is that its derivatives can be calculated directly using differentiation formulae instead of repeatedly using the differential quotient rule. The $n$th derivative of the rational interpolating function $p_{N}(x)$ evaluated at the point $x_{j}$ can be expressed in the following form:

$$
\begin{aligned}
p_{N}^{(n)}\left(x_{j}\right) & =\sum_{k=0}^{N} \frac{\mathrm{d}^{n}}{\mathrm{~d} x^{n}}\left(\frac{\left(\omega_{k} / x-x_{k}\right) u\left(x_{k}\right)}{\sum_{l=0}^{N}\left(\omega_{l} / x-x_{l}\right)}\right)_{x=x_{j}} \\
& =\sum_{k=0}^{N} D_{j k}^{(n)} u\left(x_{k}\right), \quad j=0,1, \ldots, N,
\end{aligned}
$$

where $D_{j k}^{(n)}$ is the entry of the $n$th order differentiation matrix. The entries of the first- and second-order differentiation matrices are given as follows [15]: 


$$
D_{j k}^{(1)}= \begin{cases}\frac{\omega_{k}}{\omega_{j}\left(x_{j}-x_{k}\right)} & j \neq k, \\
-\sum_{i \neq k} D_{j i}^{(1)}, j=k, & \\
D_{j k}^{(2)}=\left\{\begin{array}{l}
2 D_{j k}^{(1)}\left(D_{j j}^{(1)}-\frac{1}{x_{j}-x_{k}}\right), \quad j \neq k, \\
-\sum_{i \neq k} D_{j i}^{(2)}, j=k .
\end{array}\right.\end{cases}
$$

Note that differentiation matrices (33) only rely on weights $\omega_{k}$ and the points $x_{k}$, which is the reason why the underlying equation does not need to be converted to new coordinates after maps.

2.3. The Sinh Transform. To approximate the rapid changes in the boundary layer region, Tee and Trefethen have constructed the conformal map [17]:

$$
g_{\lambda, \delta}(x)=\lambda+\mu \sinh \left[\left(\sinh h^{-1}\left(\frac{1-\lambda}{\mu}\right)+\sinh h^{-1}\left(\frac{1+\lambda}{\mu}\right)\right) \frac{x-1}{2}+\sinh h^{-1}\left(\frac{1-\lambda}{\mu}\right)\right],
$$

where $\lambda, \mu$ are the location and width of the boundary layers, respectively. The transformed Chebyshev points $\left\{g_{\lambda, \delta}\left(x_{k}\right)\right\}_{k=0}^{N}$ are clustered near the location of boundary layer $x=\lambda$, and the density is determined by the width of the boundary layer.

To better distinguish the singular perturbation problem with two boundary layers, Tee proposed the combined sinh transform as

$$
\tilde{g}_{\mu}(x)= \begin{cases}\frac{1}{2}\left[g_{-1, \mu}(2 x+1)-1\right], & x \in[-1,0), \\ \frac{1}{2}\left[g_{1, \mu}(2 x-1)+1\right], & x \in[0,1] .\end{cases}
$$

All derivatives of the piecewise map $\tilde{g}$ at $x=0$ are continuous to preserve the spectral accuracy. For the reaction-diffusion type, the parameter in (35) should be chosen as $\mu=2 \tau_{0}$.

\section{The Rational Spectral Collocation with a Singularly-Separated Method}

The implementation and error estimation of RSC-SSM for a singularly perturbed reaction-diffusion problem with a discontinuous source term is elaborated in this section.

3.1. The Singularity-Separated Technique. Consider the problem (1). Taking the left subproblem (19) as an example, the efficient numerical method can be constructed. The homogenous equation $L^{-} u=0$ has two eigenvalues:

$$
\begin{aligned}
& \lambda_{1}=\sqrt{\frac{c}{\varepsilon}}, \\
& \lambda_{2}=-\sqrt{\frac{c}{\varepsilon}} .
\end{aligned}
$$
lution is

Let $u_{0}$ be a special solution of $L^{-} u=f$; its general so-

$$
\begin{aligned}
u(x) & =u_{0}(x)+C_{1} \phi_{1}(x)+C_{2} \phi_{2}(x), \\
\phi_{1}(x) & =e^{\lambda_{1}(x-d)}, \phi_{2}(x)=e^{\lambda_{2} x} .
\end{aligned}
$$

Note that if $\varepsilon=10^{-k}$ and $k \geq 4, \phi_{1}(1)=\phi_{2}(0)=$ $e^{\lambda_{2}} \approx e^{(-1 / \sqrt{\epsilon})} \geq e^{-10^{2}} \approx 10^{-44}=0$ can be neglected.

Thus, the solution $u_{l}(x)$ of (19) can be decomposed into two parts: $u_{l}(x)=\omega_{l}(x)+v_{l}(x)$, in which $\omega_{l}(x)=u_{0}(x)$ is the regular term and $v_{l}(x)=C_{1} \phi_{1}(x)+C_{2} \phi_{2}(x)$ is the singular term. The regular term $\omega_{l}(x)$ is the solution of an auxiliary boundary value problem:

$$
\left\{\begin{array}{l}
L^{-} \omega_{l}=-\varepsilon \omega_{l}^{\prime \prime}+c \omega_{l}=f^{-}(x), \\
\omega_{l}(0)=\frac{f^{-}(0)}{c} \\
\omega_{l}(d)=\frac{f^{-}(d)}{c}
\end{array}\right.
$$

It is known from the boundary conditions of equation (38) that $\varepsilon \omega_{l}^{\prime \prime}(0)=\varepsilon \omega_{l}^{\prime \prime}(d)=0$. The singularities of the solution are weakened.

Similarly, the solution $u_{r}(x)$ of (20) can also be decomposed into two parts: $u_{r}(x)=\omega_{r}(x)+v_{r}(x)$. The regular term $\omega_{r}(x)$ is the solution of an auxiliary boundary value problem: 


$$
\left\{\begin{array}{l}
L^{+} \omega_{r}=-\varepsilon \omega_{r}^{\prime \prime}+c \omega_{r}=f^{+}(x), \\
\omega_{r}(d)=\frac{f^{+}(d)}{c}, \\
\omega_{r}(1)=\frac{f^{+}(1)}{c} .
\end{array}\right.
$$

In the same way, $\varepsilon \omega_{r}^{\prime \prime}(d)=\varepsilon \omega_{r}^{\prime \prime}(1)=0$.

Theorem 5. The singular components $v_{l}(x), v_{r}(x)$ of (19) and (20) are

$$
\left\{\begin{array}{l}
v_{l}(x)=C_{1}^{l} e^{-\sqrt{(c / \varepsilon)} x}+C_{2}^{l} e^{\sqrt{(c / \varepsilon)}(x-d)}, \\
v_{r}(x)=C_{1}^{r} e^{-\sqrt{(c / \varepsilon)}(x-d)}+C_{2}^{r} e^{\sqrt{(c / \varepsilon)}(x-1)},
\end{array}\right.
$$

where

$$
\left\{\begin{array}{l}
C_{1}^{l}=\alpha-\frac{f^{-}(0)}{c}, \\
C_{2}^{l}=\frac{1}{2 c}\left(\sqrt{(\varepsilon / c)}\left[f^{\prime}\right](d)+[f](d)\right), \\
C_{1}^{r}=\frac{1}{2 c}\left(\sqrt{(\varepsilon / c)}\left[f^{\prime}\right](d)-[f](d)\right), \\
C_{2}^{r}=\beta-\frac{f^{+}(1)}{c},
\end{array}\right.
$$

where $w_{l}(x)$ and $w_{r}(x)$ are the solutions of the auxiliary in (38) and (39).

Proof. According to the boundary condition of (1), it is known that

$$
\left\{\begin{array}{l}
\alpha=w_{l}(0)+C_{1}^{l} \\
\beta=w_{r}(1)+C_{2}^{r} .
\end{array}\right.
$$

We can obtain $C_{1}^{l}=\alpha-\left(f^{-}(0) / c\right), C_{2}^{r}=\beta-\left(f^{+}(1) / c\right)$. On the contrary, the solution of equation (1) $u(x) \in C^{1}(\bar{\Omega})$, which is $u\left(d^{-}\right)=\left(d^{+}\right), u^{\prime}\left(d^{-}\right)=u^{\prime}\left(d^{+}\right)$. Thus, we have

$$
\left\{\begin{array}{l}
w_{l}\left(d^{-}\right)+C_{2}^{l}=w_{r}\left(d^{+}\right)+C_{1}^{r}, \\
w_{l}^{\prime}\left(d^{-}\right)+C_{2}^{l} \sqrt{(c / \varepsilon)}=w_{r}^{\prime}\left(d^{+}\right)-C_{1}^{r} \sqrt{(c / \varepsilon)} .
\end{array}\right.
$$

Furthermore,

$$
\begin{aligned}
& w_{r}\left(d^{+}\right)-w_{l}\left(d^{-}\right)=\frac{f^{+}\left(d^{+}\right)}{c}-\frac{f^{-}\left(d^{-}\right)}{c}=\frac{[f](d)}{c}, \\
& w_{r}^{\prime}\left(d^{+}\right)-w_{l}^{\prime}\left(d^{-}\right)=\frac{f^{\prime^{+}}\left(d^{+}\right)}{c}-\frac{f^{\prime^{-}}\left(d^{-}\right)}{c}=\frac{\left[f^{\prime}\right](d)}{c} .
\end{aligned}
$$

Thus, the parameters can be obtained:

$$
\left\{\begin{array}{l}
C_{2}^{l}=\frac{1}{2 c}\left(\sqrt{(\varepsilon / c)}\left[f^{\prime}\right](d)+[f](d)\right), \\
C_{1}^{r}=\frac{1}{2 c}\left(\sqrt{(\varepsilon / c)}\left[f^{\prime}\right](d)-[f](d)\right) .
\end{array}\right.
$$

Thus, the solution of the singularly-separated technique can be obtained as

$$
u_{s s}(x)= \begin{cases}\omega_{l}(x)+v_{l}(x), & x \in \Omega^{-}, \\ \omega_{r}(x)+v_{r}(x), & x \in \Omega^{+},\end{cases}
$$

where the regular terms $w_{l}(x)$ and $w_{r}(x)$ are the solutions of the auxiliary in (38) and (39) and the singular terms $v_{l}(x)$ and $v_{r}(x)$ are defined as (40).

3.2. RSC-SSM Method. The key to the success of the RSCSSM is obtaining a high-precision numerical solution for weakened singular perturbation problems (38). The rational spectral collocation with a sinh transform is selected.

First, by introducing the linear transform $x=(d / 2)(t+$ 1), $x \in[0, d], t \in[-1,1]$ and defining a new function $\widehat{u}(t)=u(x)=u((d / 2)(t+1)), \quad$ then $u^{\prime}(x)=(2 / d) \widehat{u}^{\prime}(t), u^{\prime \prime}(x)=\left(4 / d^{2}\right) \widehat{u}^{\prime \prime}(t)$, and we can rewrite (38) as

$$
\begin{aligned}
& \left\{L \widehat{\omega}=-\frac{4}{d^{2}} \varepsilon \widehat{\omega}_{l}^{\prime \prime}(t)+c \widehat{\omega}_{l}(t)=\widehat{f}^{-}(t), \quad t \in(-1,1), \widehat{\omega}_{l}(-1)=\frac{\widehat{f}^{-}(-1)}{c}, \widehat{\omega}_{l}(1)=\frac{\widehat{f}^{-}(1)}{c} .\right. \\
& -\frac{4}{d^{2}} \varepsilon D^{(2)} W_{l}+c E_{N+1} W_{l}=F,
\end{aligned}
$$

where

$$
\left\{\widehat{x}_{k}\right\}_{k=0}^{N}=\left\{g_{\delta}\left(-\cos \left(\frac{k \pi}{N}\right)\right)\right\}_{k=0}^{N},
$$

where $g$ is shown in (35).

Evaluating (47) at points $\left\{\hat{x}_{k}\right\}_{k=0}^{N}$ yields

$$
\begin{aligned}
W_{l} & =\left[w_{0}, w_{1}, \ldots, w_{N}\right]^{T}, \text { with } w_{k} \text { evaluating } w\left(t_{k}\right), \\
F & =\left[\widehat{f}_{0}, \widehat{f}_{1}, \ldots, \widehat{f}_{N}\right]^{T}, \text { with } \widehat{f}_{k} \text { evaluating } \widehat{f}\left(t_{k}\right),
\end{aligned}
$$

and $E_{N+1}$ is a unit matrix of $N+1$-order.

Let $A=-\left(4 / d^{2}\right) \varepsilon D^{(2)}+c E_{N+1}$, then equation (49) can be rewritten as 


$$
A W_{l}=F .
$$

The boundary conditions in (47) suggest that

$$
W_{l}(1)=\frac{\hat{f}^{-}(-1)}{c}, W_{l}(N+1)=\frac{\hat{f}^{-}(1)}{c} .
$$

The Dirichlet boundary conditions are imposed on the first and last rows of $A$. We substitute elements in these rows with zeros. The first zero in the first row and the last zero in the last row are replaced by 1 . Simultaneously, the first and last elements of vector $F$ are replaced by $\left(f^{-}(-1) / c\right)$ and $\left(f^{-}(1) / c\right)$.

By solving the linear algebra system of (51), we can obtain the numerical solution $W_{l}$, which is the regular term in (35). Similarly, the numerical solution $W_{r}$, which is the solution of (47), can be obtained.

According to the boundary condition of the original problem (1) and the value of the regular components $\omega_{l}$ and $\omega_{r}$, the parameters of singular terms (40) are obtained.

The numerical solution of equation (1) can be obtained as

$$
u^{N}(x)= \begin{cases}W_{l}(x)+v_{l}(x), & x \in \Omega^{-} \\ W_{r}(x)+v_{r}(x), & x \in \Omega^{+}\end{cases}
$$

3.3. Error Analysis. In this section, we establish the error estimates of the RSC-SSM (53).

Theorem 6. Let $u\left(x_{i}\right)$ and $u^{N}\left(x_{i}\right)$ be the exact solution and numerical solution of the original problem (1), respectively. Then, we have

$$
\left\|u-u^{N}\right\| \leq c_{1} e^{-(\sqrt{c} / \sqrt{\varepsilon})}+c_{2} \rho^{-N} .
$$

Proof. Let $z=u-u^{N}$; then, the error $z$ satisfies

$$
\begin{aligned}
\|z\| & =\left\|u-u^{N}\right\|=\left\|u-u_{S S}+u_{S S}-u^{N}\right\| \\
& \leq\left\|u-u_{S S}\right\|+\left\|u_{S S}-u^{N}\right\| \\
& =\left\|u-u_{S S}\right\|+\max \left\{\left\|\omega_{l}-W_{l}\right\|,\left\|\omega_{r}-W_{r}\right\|\right\} .
\end{aligned}
$$

Since the singular correct function $v(x)$ is the solution of the homogenous equations $L v=0$, set $R=u-u_{S S}$, then

$$
\begin{aligned}
\mathrm{LR} & =L\left(u-u_{S S}\right)=L(u-\omega-v) \\
& =-\varepsilon u^{\prime \prime}+c u-\left(-\varepsilon \omega^{\prime \prime}+c \omega\right)-L v=0,
\end{aligned}
$$

and, according to the proof of Theorem 5, we have

$$
\begin{aligned}
& {[R](d) \approx\left(u\left(d^{+}\right)-w_{r}\left(d^{+}\right)-C_{1}^{r}\right)-\left(u\left(d^{-}\right)-w_{l}\left(d^{-}\right)-C_{2}^{l}\right)=0,} \\
& {\left[R^{\prime}\right](d) \approx\left(u^{\prime}\left(d^{+}\right)-w_{r}^{\prime}\left(d^{+}\right)+\sqrt{\frac{c}{\varepsilon}} C_{1}^{r}\right)-\left(u^{\prime}\left(d^{-}\right)-w_{l}^{\prime}\left(d^{-}\right)-\sqrt{\frac{c}{\varepsilon}} C_{2}^{l}\right)=0 .}
\end{aligned}
$$

Simultaneously,

$$
\begin{aligned}
& R(0)=u(0)-\omega_{l}(0)-v_{l}(0)=\alpha-\frac{f^{-}(0)}{c}-C_{1}^{l}-C_{2}^{l} e^{-d \sqrt{(c / \varepsilon)}}=-C_{2}^{l} e^{-d \sqrt{(c / \varepsilon)}}, \\
& R(1)=u(1)-\omega_{r}(1)-v_{r}(1)=\beta-\frac{f^{+}(1)}{c}-C_{1}^{r} e^{-(1-d) \sqrt{(c / \varepsilon)}}-C_{2}^{r}=-C_{1}^{r} e^{(d-1) \sqrt{(c / \varepsilon)}} .
\end{aligned}
$$

According to Lemma 2, we can obtain

$$
\begin{aligned}
\|R\| & =\left\|u-u_{S S}\right\| \leq \max \{|R(0)|,|R(1)|\} \\
& =\max \left\{\left|C_{2}^{l} e^{-d \sqrt{(c / \varepsilon)}}\right|,\left|C_{1}^{r} e^{(d-1) \sqrt{(c / \varepsilon)}}\right|\right\} \\
& \leq \frac{1}{2 c}\left(|[f](d)|+\left|\left[f^{\prime}\right](d)\right|\right) e^{-\sqrt{(c / \varepsilon)}} .
\end{aligned}
$$

A combination of the convergence of rational spectral collocation (31) leads to

$$
\left\|u_{S S}-u^{N}\right\|=\max \left\{\left\|\omega_{l}-W_{l}\right\|,\left\|\omega_{r}-W_{r}\right\|\right\} \leq c_{2} \rho^{-N} .
$$

By considering (59) and (60), it leads to

$$
\left\|u-u^{N}\right\| \leq\left\|u-u_{S S}\right\|+\left\|u_{S S}-u^{N}\right\| \leq c_{1} e^{-(\sqrt{c} / \sqrt{\varepsilon})}+c_{2} \rho^{-N} \text {. }
$$

Thus, we can establish a rational spectral method with a singularity-separated technique that can almost achieve spectral accuracy.

\section{Numerical Experiments}

To demonstrate the accuracy and efficiency of RSC-SSM, we will use the novel method to solve a singularly perturbed reaction-diffusion problem discontinuous source term and 
TABle 1: Maximum pointwise errors for RSC-SSM and finite different schemes $[9,18]$.

\begin{tabular}{|c|c|c|c|c|c|c|}
\hline \multirow{2}{*}{$(\varepsilon / N)$} & \multirow{2}{*}{$\begin{array}{c}\text { RSC-SSM } \\
32\end{array}$} & \multicolumn{2}{|c|}{ Finite difference [9] } & \multicolumn{3}{|c|}{ Hybrid scheme [18] } \\
\hline & & 64 & 128 & 32 & 64 & 128 \\
\hline $1 e-2$ & $1.07 e-13$ & $3.49 e-04$ & $1.77 e-04$ & $*$ & $*$ & $*$ \\
\hline $1 e-3$ & $2.19 e-14$ & $7.30 e-03$ & $9.42 e-04$ & $*$ & $*$ & $*$ \\
\hline $1 e-4$ & $3.61 e-15$ & $7.30 e-03$ & $9.42 e-04$ & $*$ & $*$ & $*$ \\
\hline $1 e-6$ & $3.11 e-15$ & $7.30 e-03$ & $9.42 e-04$ & $*$ & $*$ & $*$ \\
\hline $2^{-4}$ & $6.75 e-02$ & * & * & $1.72 e-03$ & $8.25 e-04$ & $4.05 e-04$ \\
\hline $2^{-8}$ & $1.66 e-04$ & $*$ & * & $6.45 e-04$ & $2.49 e-04$ & $1.08 e-04$ \\
\hline $2^{-12}$ & $1.00 e-13$ & $*$ & * & $2.69 e-03$ & $1.73 e-03$ & $6.89 e-04$ \\
\hline $2^{-16}$ & $4.95 e-14$ & $*$ & $*$ & $4.29 e-03$ & $2.04 e-03$ & $9.95 e-04$ \\
\hline $2^{-20}$ & $1.78 e-14$ & * & * & $7.23 e-03$ & $2.52 e-03$ & $1.04 e-03$ \\
\hline
\end{tabular}

${ }^{*}$ References $[9,18]$ do not compute.

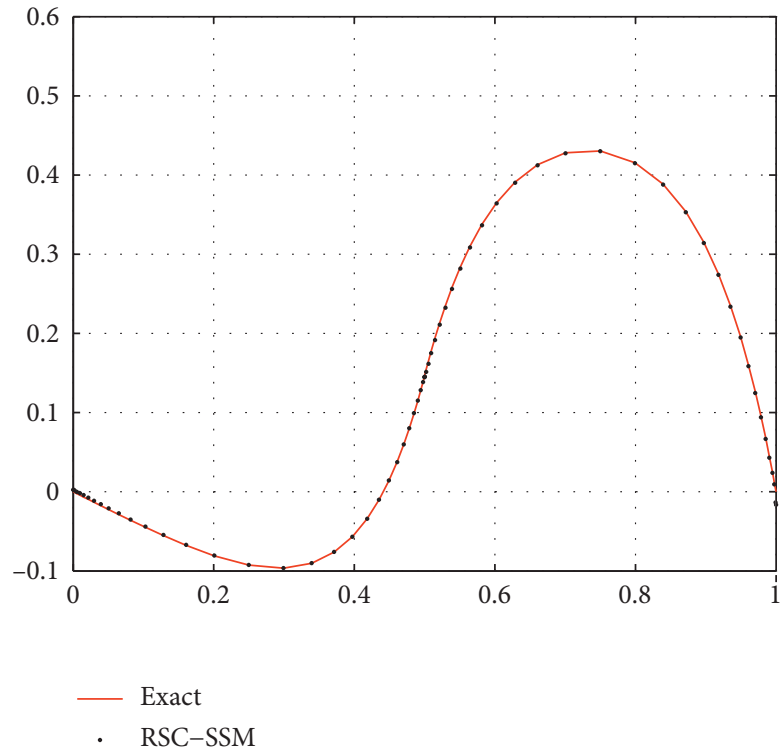

(a)

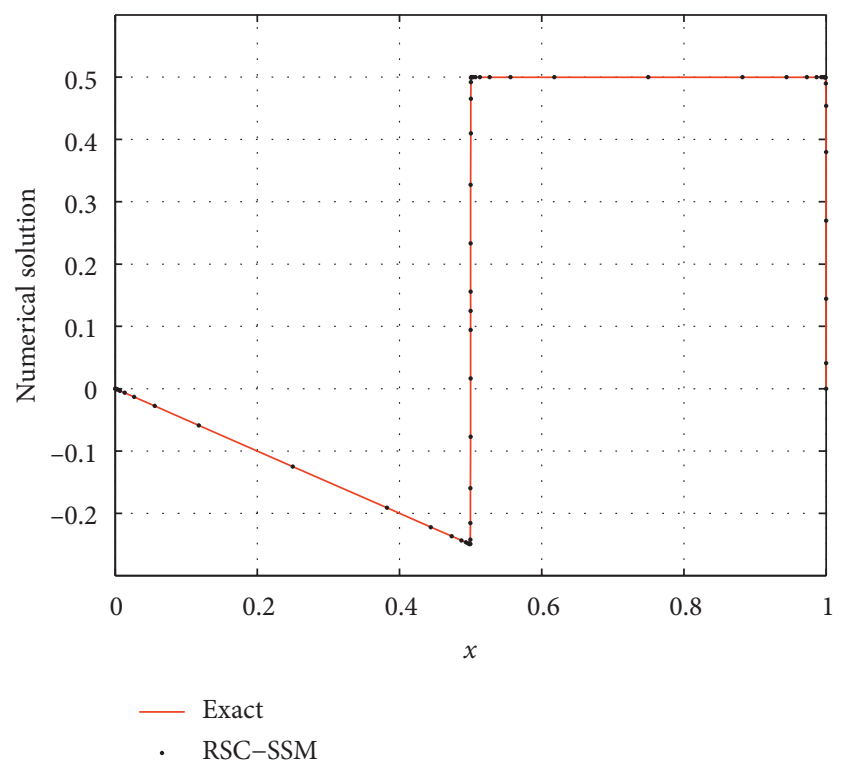

(b)

Figure 1: Numerical solution vs. exact solution in Example $(N=32)$ : (a) $\varepsilon=10^{-1}$; (b) $\varepsilon=10^{-4}$.

compare the results with other existing methods. We verify the theoretical results obtained in the previous section through numerical experiments. The maximum relative errors of the solution are given by

$$
e=\frac{\left\|u_{N}-u\right\|_{\infty}}{\left\|u_{E}\right\|_{\infty}},
$$

where $u_{N}$ and $u$ are the numerical and exact solutions, respectively.

In our computations, all experiments are carried out using MATLAB (version R2014a) on a PC with a $2.5 \mathrm{GHz}$ central processing unit (Intel Core i5-2450M), 4.00 GB memory, and Windows 7 operating system. 


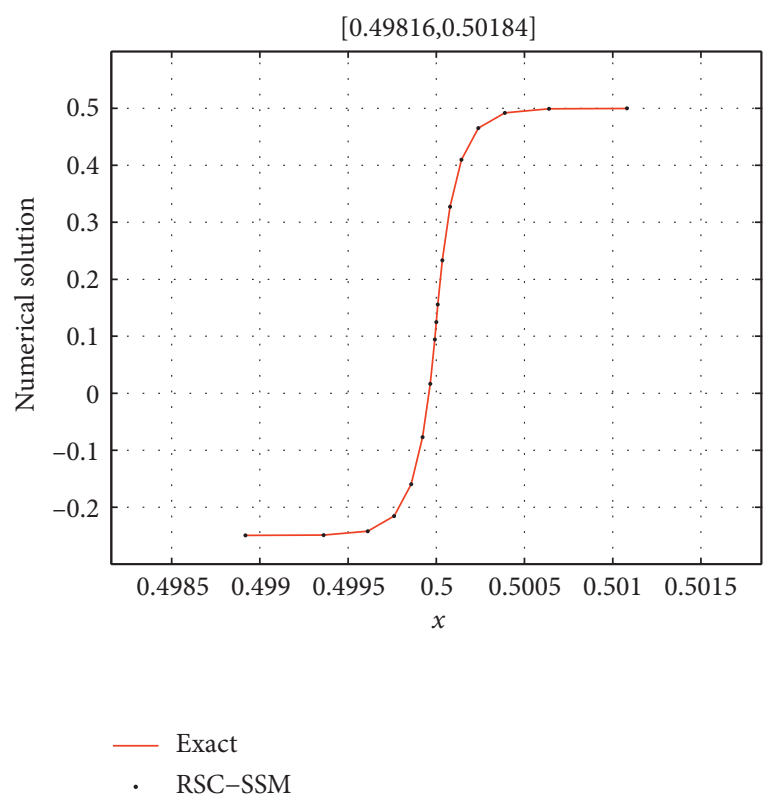

(a)

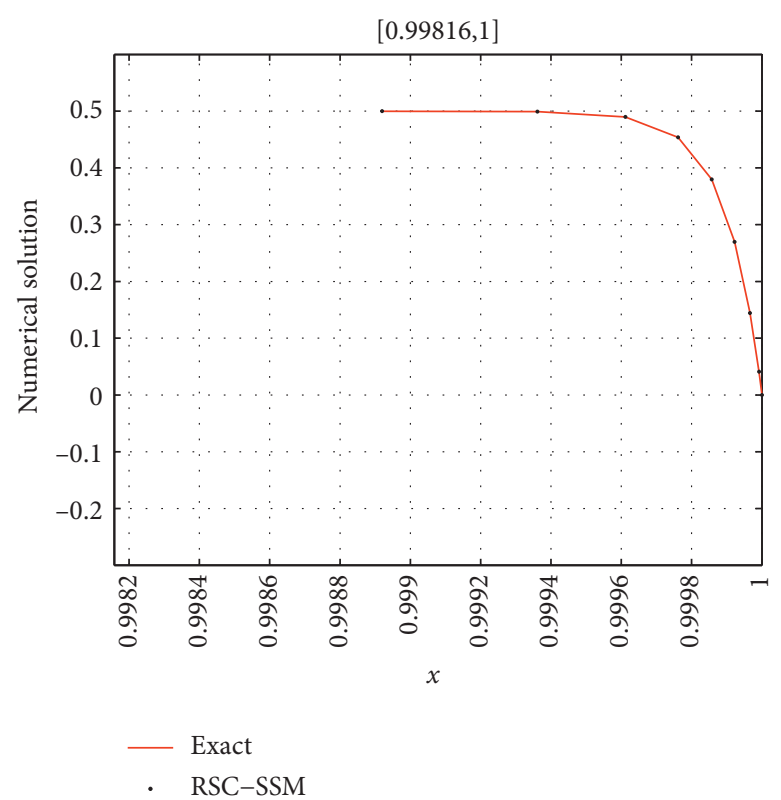

(b)

FIGURE 2: Numerical solution vs. exact solution in Example $\left(\varepsilon=10^{-4}, N=32\right)$ : (a) interior layer region; (b) boundary layer region.

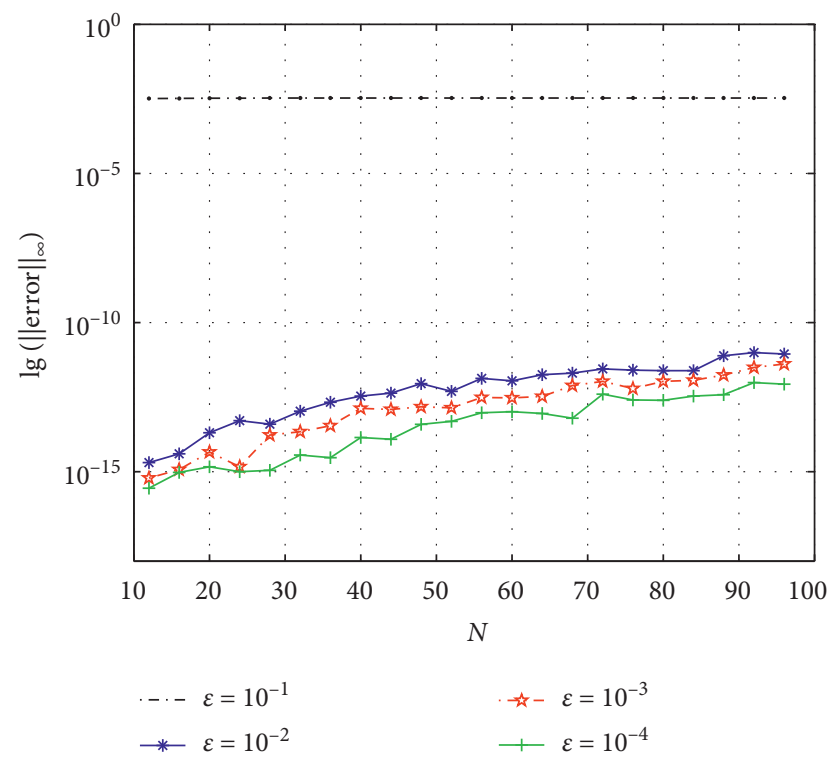

FIgURE 3: The convergence rate.

Example. Consider the following singularly perturbed problem with discontinuous source term:

$$
\begin{aligned}
-\varepsilon^{2} u^{\prime \prime}(x)+u(x) & =f(x), \quad x \in \Omega^{-} \cup \Omega^{+}, \\
u(0) & =y(1)=f(0),
\end{aligned}
$$

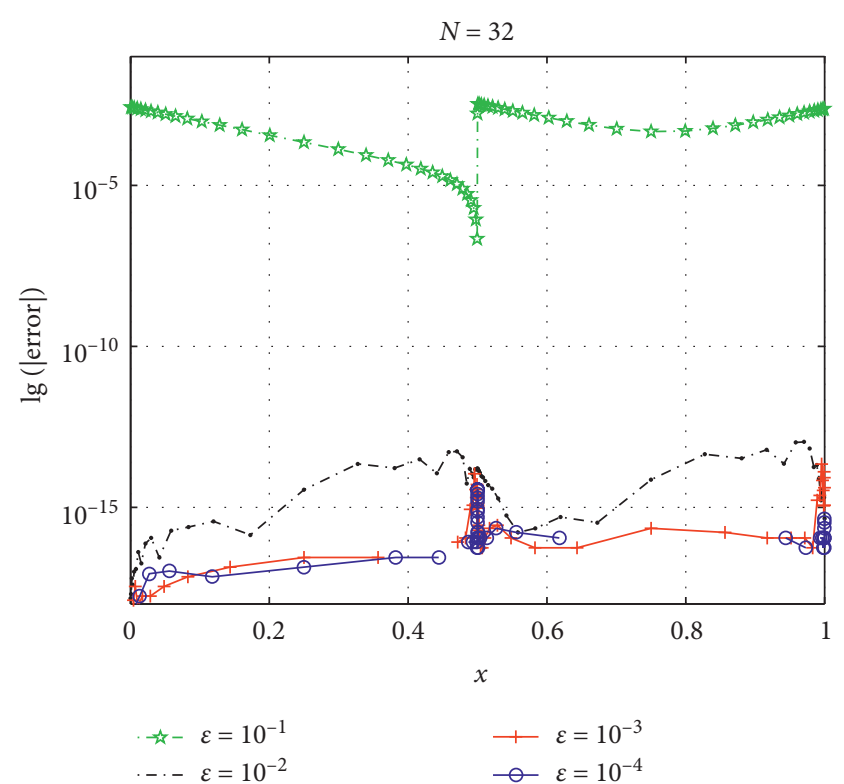

FIgURE 4: The pointwise error $(N=32)$.

where

$$
f(x)= \begin{cases}-0.5 x, & 0 \leq x \leq 0.5 \\ 0.5, & 0.5<x \leq 1\end{cases}
$$


The exact solution of this problem can be expressed as

$$
u(x)= \begin{cases}0.25(A+B)\left(e^{-(0.5-x) / \varepsilon}-e^{-(0.5+x) / \varepsilon}\right)-0.5 x, & 0 \leq x \leq 0.5, \\ 0.25(A-B)\left(e^{(1-2 x) / 2 \varepsilon}-e^{(2 x-3) / 2 \varepsilon}\right)+0.5\left(1-e^{-(1-x) / \varepsilon}\right), & 0.5<x \leq 1,\end{cases}
$$

where $A$ and $B$ are constants related to $\varepsilon$ :

$$
\begin{aligned}
& A=\frac{\varepsilon-e^{-(1 / 2 \varepsilon)}}{1+e^{-(1 / \varepsilon)}}, \\
& B=\frac{1.5-e^{-(1 / 2 \varepsilon)}}{1-e^{-(1 / \varepsilon)}} .
\end{aligned}
$$

Obviously, there is an interior layer at $x=0.5$ in the solution.

In the RSC-SSM method, the parameters in combined transform of (35) are chosen as $\mu=-4 \varepsilon \ln \varepsilon$.

The RSC-SSM method and the different schemes in $[9,18]$ are used to solve this problem with several choices of $N$ and $\varepsilon$. The maximum relative errors of the three methods are listed in Table 1.

Compared with the existing difference schemes $[9,18]$, the RSC-SSM accuracy has been significantly improved for the case with $\varepsilon^{2} \leq 10^{-3}$. However, when the parameters $\varepsilon^{2} \geq 10^{-2}$, the accuracy of the RSC-SSM is similar to that of the difference method. This is because that the discarded item reached $e^{-(1 / \varepsilon)}=e^{-10} \approx 4.5 \times 10^{-5}$ in singularly-separated technique. In Table 1, we investigate the effects of the singular perturbation parameter $\varepsilon$ on the errors. We can see that a decrease in $\varepsilon$ reduces the maximum relative errors.

Figure 1 depicts the numerical solution and the exact solution of the whole region with different $\varepsilon$. The numerical solution and exact solutions in both the interior and the boundary layers are given in Figure 2.

Figure 3 shows the maximum relative errors in the semilog scale with $\varepsilon=10^{-1}, 10^{-2}, 10^{-3}$, and $10^{-4}$, respectively. It demonstrates the influence of points $N$ on the errors. The convergence rates are almost balanced, and even the errors have increased due to the cumulative effects with an increase in $N$. Figure 4 shows the pointwise errors of the function in the whole region with $\varepsilon=10^{-1}, 10^{-2}, 10^{-3}$, and $10^{-4}$, respectively. The smaller the parameter $\varepsilon$ is, the more accurate the RSC-SSM result. Figures 3 and 4 also show that the error mainly comes from the discarded items in the process of singular separation. This confirms the results discussed in the previous section.

\section{Conclusions}

In this paper, a novel numerical method, named RSC-SSM, has been proposed to solve singularly perturbed boundary value problems with discontinuous source term. The solution is composed of the weaker singularity auxiliary solution and a singular correct function. The numerical experiment illustrates that the proposed RSC-SSM is obviously a better choice to other existing numerical methods available for the singularly perturbed boundary value problems with discontinuous source term. The numerical results demonstrate the almost spectral accuracy of the proposed algorithm and coincide with the theoretical analysis. Moreover, the theoretical and numerical frameworks presented in this paper can be extended to more complex problems.

\section{Data Availability}

The data used to support the findings of this study are included in the article.

\section{Conflicts of Interest}

The author declares that there are no conflicts of interest regarding the publication of this paper.

\section{Acknowledgments}

The author is grateful to Prof. Y J Wu for his valuable suggestions and help for the work related to this paper. The work was partially supported by the Research Project of Science and Technology of Colleges and Universities in Ningxia (no. NGY2020056), the First-Class Disciplines Foundation of Ningxia (no. NXYLXK2017B09), and the Science Foundation of North Minzu University (no. 2019XYZSX04).

\section{References}

[1] M. Kumar and N. Singh, "A collection of computational techniques for solving singular boundary-value problems," Advances in Engineering Software, vol. 40, no. 4, pp. 288-297, 2009.

[2] M. K. Kadalbajoo and V. Gupta, "A brief survey on numerical methods for solving singularly perturbed problems," Applied Mathematics and Computation, vol. 217, no. 8, pp. 3641-3716, 2010.

[3] J. J. H. Miller, E. O’Riordan, and G. I. Shishkin, Fitted Numerical Methods for Singular Perturbation Problems: Error Estimates in the Maximum Norm for Linear Problems in One and Two Dimensions, World Scientific Publishing Co. Pte. Ltd, Singapore, 1996.

[4] M. Chandru and V. Shanthi, "A boundary value technique for singularly perturbed boundary value problem of reactiondiffusion with non-smooth data," Journal of Engineering Science and Technology, vol. 9, pp. 32-45, 2014.

[5] M. Chandru, T. Prabha, and V. Shanthi, "A hybrid difference scheme for a second-order singularly perturbed reactiondiffusion problem with non-smooth data," International Journal of Applied and Computational Mathematics, vol. 1, no. 1, pp. 87-100, 2015.

[6] H. G. Roos and H. Zarin, "A second-order scheme for singularly perturbed differential equations with discontinuous 
source term," Journal of Numerical Mathematics, vol. 10, pp. 275-289, 2002.

[7] P. A. Farrell, E. O’Riordan, and G. I. Shishkin, "A class of singularly perturbed semilinear differential equations with interior layers," Mathematics of Computation, vol. 74, no. 252, pp. 1759-1777, 2005.

[8] C. de Falco and E. O'Riordan, "Interior layers in a reactiondiffusion equation with a discontinuous diffusion coefficient," International Journal of Numerical Analysis and Modeling, vol. 7, pp. 444-461, 2010.

[9] Z. Cen, A. Le, and A. Xu, "A high-order finite difference scheme for a singularly perturbed reaction-diffusion problem with an interior layer," Advances in Difference Equations, vol. 202, pp. 1-8, 2017.

[10] J.-P. Berrut, R. Baltensperger, and H. D. Mittelmann, "Recent developments in barycentric rational interpolation," Trends and Applications in Constructive Approximation, vol. 15, pp. 27-51, 2005.

[11] S. Q. Chen and Y. W. Wang, "A rational spectral collocation method for third-order singularly perturbed problems," Journal of Computational and Applied Mathematics, vol. 307, pp. 93-105, 2016.

[12] S. Q. Chen, Y. Wei Wang, and X. Wu, "Rational spectral collocation method for a coupled system of singularly perturbed boundary valus problems," Journal of Computational Mathematics, vol. 29, no. 4, pp. 458-473, 2011.

[13] C. M. Chen and J. Yang, "The singularity-separated method for the singular perturbation problems in 1-d," International Journal of Numerical Analysis and Modeling, vol. 15, no. 1-2, pp. 102-110, 2017.

[14] L. Yang, "Rational spectral collocation combined with the singularity separated method for a system of singularly perturbed boundary value problems," Mathematical Problems in Engineering, vol. 2019, no. 4, pp. 1-12, 2019.

[15] J.-P. Berrut and L. N. Trefethen, "Barycentric Lagrange interpolation," SIAM Review, vol. 46, no. 3, pp. 501-517, 2004.

[16] R. Baltensperger, J.-P. Berrut, and B. Noël, "Exponential convergence of a linear rational interpolant between transformed Chebyshev points," Mathematics of Computation, vol. 68, no. 227, pp. 1109-1121, 1999.

[17] T. W. Tee and L. N. Trefethen, "A rational spectral collocation method with adaptively transformed Chebyshev grid points," SIAM Journal on Scientific Computing, vol. 28, no. 5, pp. 1798-1811, 2006.

[18] J. J. H. Miller, E. O’Riordan, G. I. Shishkin, and S. Wang, “A parameter-uniform Schwarz method for a singularly perturbed reaction-diffusion problem with an interior layer," Applied Numerical Mathematics, vol. 35, no. 4, pp. 323-337, 2000. 